\title{
Characterization Of Pin Diode Silicon Radiation Detector
}

\author{
Samichi Srivastava ${ }^{1}$, Rabinber Henry ${ }^{2}$, Anita Topka $\mathbf{R}^{3}$ \\ ${ }^{1}$ PG Scholar, ${ }^{2}$ Lecturer International Institute of Information Technology, Pune - 411057 \\ ${ }^{3}$ Scientist F-Bhabha Atomic Research Centre, Trombay - 400085 \\ E-mail : samichi_120385@yahoo.co.in rabinderh@isquareit.ac.in anitatopkar@barc.gov.in
}

\section{Abstract}

Bhabha atomic research centre has been exploring some of the unique features of silicon detector in a variety of nuclear structure experiment using high-energy photons and heavy ions projectiles. Current experimental application make use of large area silicon detector with depletion thickness of $\sim 150-1000 \mu \mathrm{m}$. The low cost silicon detector shows excellent energy and position resolution. In this paper we discuss the physical mechanism involved in detector operation and one set of mask designing for the silicon radiation detector and also clarify how these effects set a limit to achievable timing performance.

Key words : PIN diode, pulse mode operation, radiation detector

\section{INTRODUCTION}

Nuclear radiation from the backbone of most of the nuclear physics and high energy particle silicon radiation detector has advantages over conventional detectors. It needs no cooling system. Ordinary silicon PIN photodiodes can serve as detectors for X-ray and gamma ray photons [6]. The detection efficiency is a function of the thickness of the silicon wafer. For a wafer thickness of $300 \mu \mathrm{m}$ (ignoring attenuation in the diode window and/or package) the detection efficiency is close to $100 \%$ at 10 $\mathrm{KeV}$, falling to approximately $1 \%$ at $150 \mathrm{KeV}$. For energies above approximately $60 \mathrm{KeV}$, photons interact almost entirely through Compton scattering. Moreover, the active region of the diode is in electronic equilibrium with the surrounding medium--the diode package, substrate, window and outer coating, etc., so that Compton recoil electrons which are produced near--and close enough to penetrate--the active volume of the diode, are also detected. For this reason the overall detection efficiency at $150 \mathrm{KeV}$ and above is maintained fairly constant (approximately 1\%) over a wide range of photon energies. Thus, a silicon PIN diode can be thought of as a solid-state equivalent to an ionization-chamber radiation detector $[1,3]$.

\section{TECHNICAL GUIDE}

\section{Benefits}

Silicon radiation detectors offer the benefit of low voltage operation combined with high sensitivity, longterm reliability and ruggedness. PlasmaAntennas is one of the worlds leading suppliers of silicon radiation detectors, manufactured under clean room conditions and extensively tested to ensure that they meet stringent performance specifications.

\section{Detector types}

The SRD is rugged diffused junction diodes and is ideal for gross alpha counting or low-resolution spectrometry. It is designed to have no electrical contacts on the front face, thus maximizing geometric efficiency. Furthermore, the rugged front face is easily cleaned with a solvent-moistened swab. Applications include environmental (e.g. actinide in air) monitoring, portable contamination monitoring instruments and filter paper counters and scanners The IRD range is fabricated using the oxidation, implantation and photolithography techniques routinely encountered in integrated circuit manufacture [.Combining high performance with ruggedness, the IRD range is suitable for a wide range of applications, including: alpha spectrometry, combined alpha/beta monitoring, low energy beta detection, back scattered electron imaging and heavy ion detection. Standard models are available in both ranges but special detectors are also manufactured to meet customers' individual. Specifications. Standard models are typically supplied in stainless steel cans and are bakeable to $100^{\circ} \mathrm{C}$. Special models bakeable to $200 \mathrm{C}$ or for use below room temperature can also be supplied. Detectors can also be supplied as bare die, in custom package, or on specially designed circuit boards $[3,5]$.

\section{CHARACTERISTICS OF SILICON RADIATION DETECTOR}

It is a p-n junction diode which is reverse biased to create a region free of charge carriers, known as the depletion region. By using high resistivity silicon, depletion depths of $300 \mu \mathrm{m}$ or more can be achieved at a bias of 50$100 \mathrm{~V}$. lonising or electromagnetic radiation entering the depletion region creates electron-hole pairs which are separated by the bias field and collected at the electrodes. The number of electron-hole pairs, and therefore the amount of charge collected, is directly proportional to the energy of the incoming radiation. The detection system comprises the diode, an external pre- amplifier and signal processing and data collection electronics. The detector 
itself may be a single $p-n$ junction, or multiple junctions arranged in arrays, quadrants, strips or pixels [2].

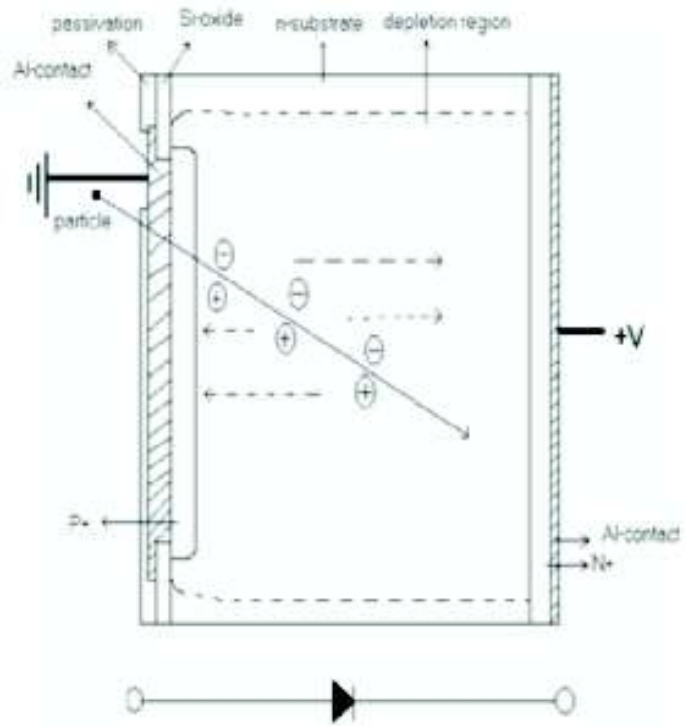

Fig. 1. Operative principle of silicon detector

The IV characterization of PIN diode detectors of area $100 \mathrm{~mm}^{2}$ circular, $100 \mathrm{~mm}^{2}$ square, $100 \mathrm{~mm}^{2}$ array has been done.

D)
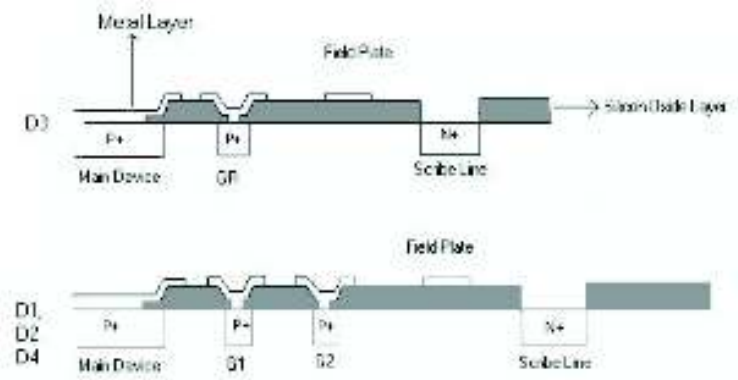

Fig.2. Cross sectional view of PIN diode detector for characterization

\section{Electrical contacts}

In order to construct practical radiation detector, some means must be provided to collect the electrical charges created by radiation at either boundary of the semiconductor detector material. An ohmic contact is nonrectifying electrode through which charge of either sign can flow freely. If the two ohmic contacts are fitted on opposite faces of slab of semiconductor and connected to detection circuit the equilibrium charge carrier concentration can be maintained. The steady state leakage currents that are observed using ohmic contacts are too high, even with highest resistivity materials the general blocking contacts are to be used. The general blocking contacts are two sides of a p-n semiconductor junction .It is very difficult to inject electron from $p$ side of this junction because holes are majority carriers. At the opposite side electrons are minority carriers and holes can not be readily injected $[1,6]$.

\section{Leakage current}

The leakage current consists of only diffusion current, but practically impurities, contaminations and process induced defects in silicon, $\mathrm{Si} / \mathrm{SiO} 2$ interface states contribute to leakage current of diodes. The resistivity of highest purity silicon currently available is about 50,000 ? $-\mathrm{cm}$. If we cut $1 \mathrm{~mm}$ thickness of silicon with surface area of $1 \mathrm{~cm} 2$, the electrical resistance between two faces will be 5000 ? . An applied voltage of $500 \mathrm{~V}$ would cause a current of $0.1 \mathrm{~A}$ through silicon. The maximum current generated by a pulse of 105 radiation will produce about 10-6A. This bulk current should be reduced because sometime leakage current becomes more significant than bulk leakage. That is why it is difficult to make a detector with leakage current less than 1 $\mathrm{nA} / \mathrm{cm} 2$. After getting exposed to radiation, the leakage current increases manifold $[1,5]$. The actual current through the diode in forward biased mode is $(I)$

$$
\text { Where } \begin{array}{ll}
I=I s[e(q V d / k T) 1] \\
I s=\text { Diffusion current } \\
q=\text { Charge of electron } \\
V d=\text { Voltage across the diode } \\
k=\text { Boltzman constant }(1.38 E-23 \text { Joules Kelvin- } 1 \\
T=\text { Absolute temperature in } \mathrm{K}
\end{array}
$$

\section{Pulse-Mode Operation}

Stable, reliable operation at low-to-medium exposure doserates in general radiation survey and operating the PIN diode detector in AC-coupled pulsemode enhances monitoring applications. This essentially eliminates drift and instability due to changes in system parameters, such as diode leakage current, with time and temperature.

In this mode of operation the diode is closely coupled to a charge-integrating preamplifier so that individual $x$-ray or gamma-ray photon interactions are detected as discrete pulses of current. The preamplifier gain, expressed in units of "volts per unit charge" is $1 /$ Cint, where Cint is the value of the integrating capacitor which, in this implementation, is of the order of $2 \times 10-12$ farads $[1,7]$.

Assume that an incident $511 \mathrm{KeV}$ photon produces a $340 \mathrm{Kev}$ recoil electron in the diode. This, in turn, produces a charge of $340,000 \times 1.6 \times 10-19 / 3.6=1.51 \times$ $10-14$ coulombs deposited in 2 picofarads, or a voltage pulse whose amplitude $=7.55$ millivolts. Individual voltage pulses are then further amplified, threshold, and 
integrated. We eliminate system noise by introducing a low-energy threshold before the input to a bipolar junction transistor - charge-pump. This, in turn, is followed by an $\mathrm{RC}$-integrating filter with a time-constant nominally $=1$ second. The overall system gain beyond the preamp is set so that a $1.33 \mathrm{mV}$ preamp-output pulse $(60 \mathrm{KeV}$ photon energy) just exceeds the threshold. The input current to the charge-pump is set by a series resistor. The output of the charge pump / filter is a DC current proportional to doserate in the detector which may be read by a meter, chart recorder, or computer data-acquisition system. In addition, a current-to-pulse-rate converter provides a TTLcompatible output for convenient interfacing to computer process-control and monitoring systems [7].

\section{Specifications for standard models}

Type

Sensitive area Operating voltage Depletion depth Window thickness Operating temperature Pressure

\section{SIMULATION OF SILICON DETECTOR}

Finalized diode detector has been simulated using SILVACO tool and process and device simulation has been carried out to check it's electrical parameters and device parameters.

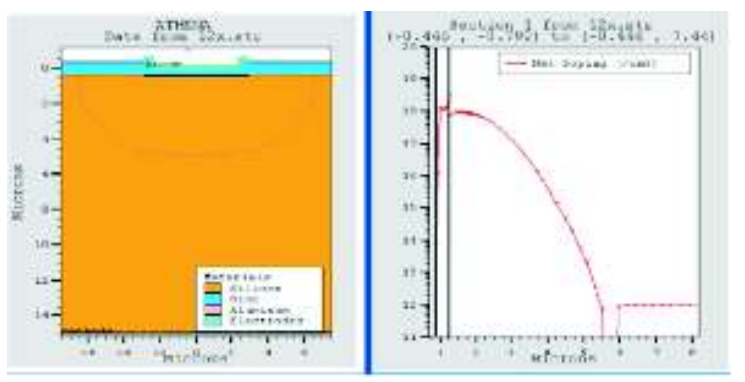

Fig. 3. Final silicon detector with it's doping profile

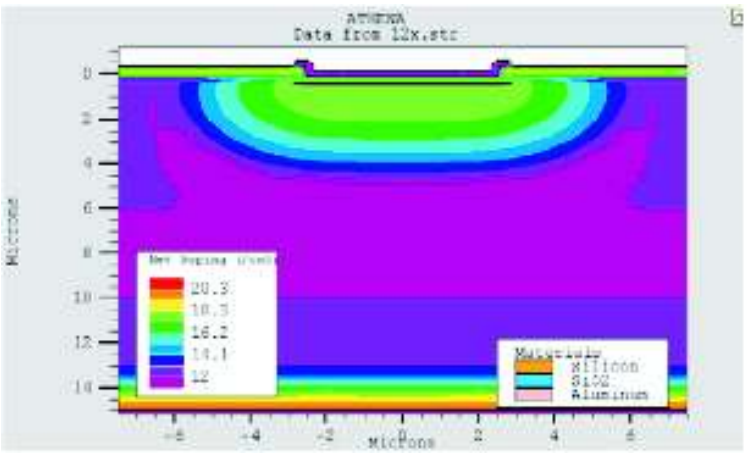

Fig. 4. Doping contour for silicon diode detector
Where some standard specification is as follows:

silicon orientation $=\langle 111\rangle$

c.phosph $=1.0 \mathrm{e} 12$

diffusion time for sacrificial oxidation $=40$ minutes

ramp up temp $=10^{\circ} \mathrm{C}$

f. $02=0.051 /$ minute

f.n2 $=51 /$ minute

initial oxidation diffusion time $=55$ minute

implant boron energy $=80$

dose $=2.0 \mathrm{e} 14$

back impant phosphorous energy $=110$ dose $=7.0 \mathrm{e} 15$

On the basis of this specification further mask has to be design, which includes a number of silicon diode detector designs of different area, parameters.

Table.1 PIN diode detectors of different area, different sizes used in Mask designing

\begin{tabular}{|c|c|c|c|}
\hline $5 \mathrm{r}$ & Ipe of $\mathrm{PIN}$ & Ars of diade deiacor & I0a! \\
\hline $\mathrm{N}_{6}$ & fiods & & \\
\hline (i) & Qusant & $100 \mathrm{mr}^{2}$ with $2 \mathrm{gx}$ of $100 \mathrm{~m}$ & 2 \\
\hline (a) & Qetase: & 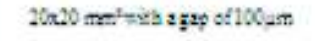 & 2 \\
\hline$\Leftrightarrow$ & Sngindodn & $25 \times 12 \mathrm{~mm}^{2}$ & : \\
\hline$\left(\mathbf{s}^{\prime}\right)$ & Aray & $1.2 \mathrm{x} 1.9 \mathrm{mr}$ vith agep of 25 un & 10 \\
\hline (⿻) & Aray & $20 \mathrm{xl}$ m: wib a gop of $1 \mathrm{~mm}$ & 5 \\
\hline$(-8)$ & Aray & 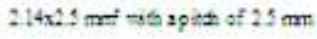 & 9 \\
\hline (G) & Ciratiar & $300 \mathrm{~mm}^{2}$ & 2 \\
\hline (vis) & Cindz & $160 \mathrm{~mm}^{2}$ & : \\
\hline (fx) & Quasan Cresta & $50 \mathrm{~mm}^{2}$ & 3 \\
\hline (8) & Cravir & $5 \mathrm{~mm}^{2}$ & 26 \\
\hline$(\mathbf{w})$ & Cirsilar & Dismetac0. $\mathrm{mm}$ & S4 \\
\hline
\end{tabular}

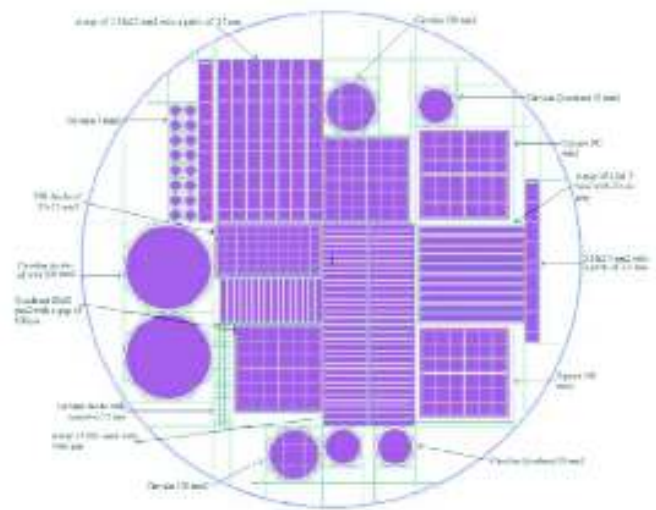

Fig. 5. Layout of all layers of mask

\section{RESULTS AND ANALYSIS}

Silicon radiation detector diode such as $100 \mathrm{~mm}^{2}$, $300 \mathrm{~mm}^{2}, 400 \mathrm{~mm}^{2}$ has been fabricated and manufactures 
which on later has been tested using the experimental setup. And also wafer level testing has been done to check potentiality of all the diode detectors on a single mask. Fig. 7. is showing the current and voltage graph for 7 PIN circular diodes of $100 \mathrm{~mm}^{2}$ that have been tested after fabrication. As it is clear that all diodes are right except one diode i.e $100 \mathrm{~mm}^{2}$ circular 2 showing with red color.

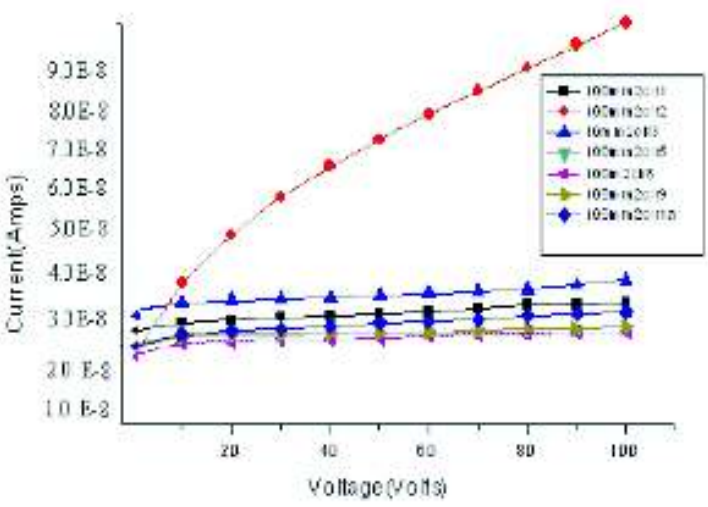

Fig. 6. IV Characteristics of $100 \mathrm{~mm}^{2}$ circular PIN diodes

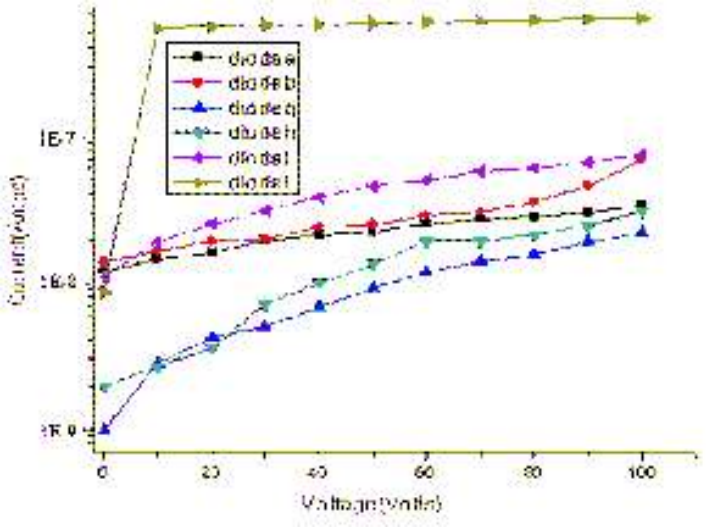

Fig. 7. IV Characteristics of $100 \mathrm{~mm}^{2}$ square PIN diodes

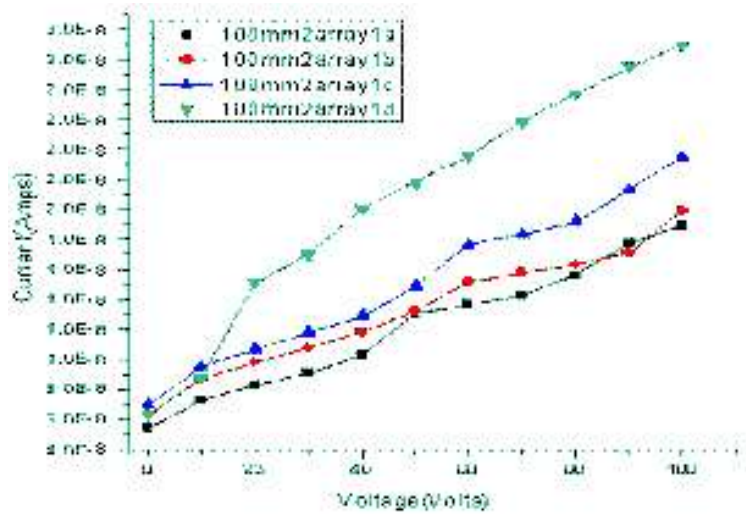

Fig. 8. IV Characteristics of $100 \mathrm{~mm}^{2}$ array PIN diodes

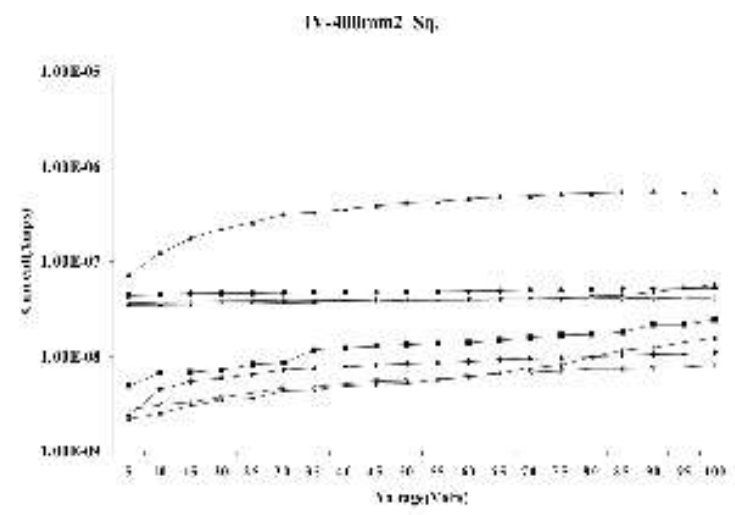

Fig.9. IV Characteristics of $300 \mathrm{~mm}^{2}$ circular PIN diode

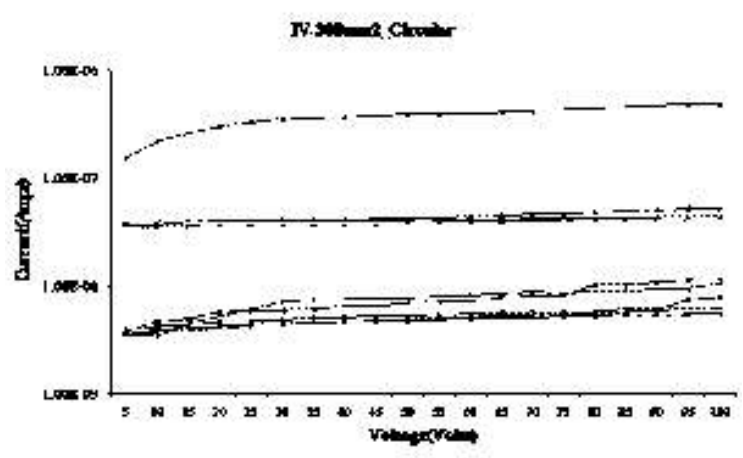

Fig.10. IV Characteristics of $400 \mathrm{~mm}^{2}$ square PIN diode

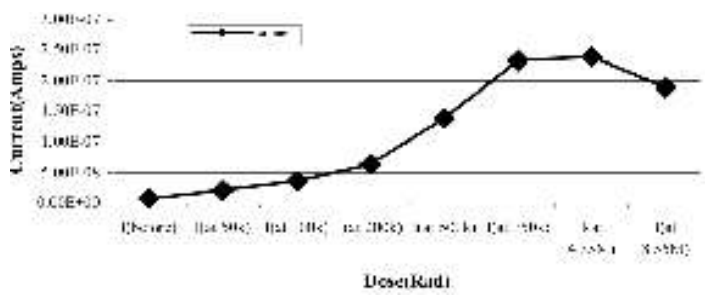

Fig.11. Leakage current @ 100V vs. radiation dose

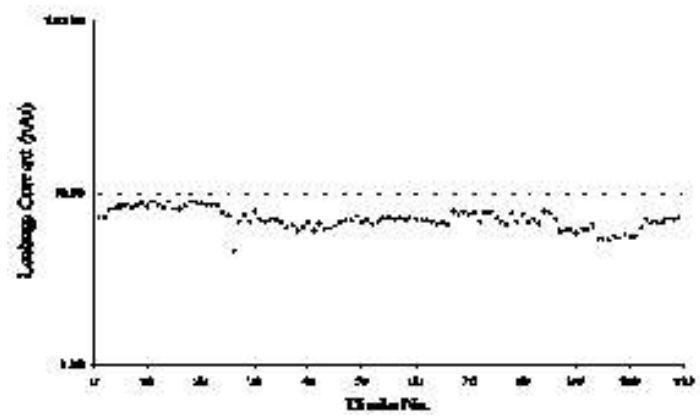

Fig. 12. Wafer level testing results showing uniformity of leakage current over the wafer 


\section{CONCLUSION}

The goal of this work is to study various types of silicon detectors specially PIN diode detectors. Process and device simulation of PIN diodes have been carried out using ATLAS and ATHENA to study process parameters for the device fabrication. IV characterization of diode detectors was also done to check their performance. Layout of various types, various shapes of PIN photo diode detector using Tanner EDA tools for the designing of mask as per the requirement has been carried out.

\section{REFERENCES}

[1] Glenn F. knoll "Radiation Detectors and Measurements" Third Edition.

[2] S.S. Kapoor, V. S. Ramamurthy "Nuclear Radiation Detectors".

[3] IEEE Transactions on Nuclear Science, October 2004, "Silicon Radiation Detectors", Vol. 51, no. 5.

[4] F.S. Goulding and R.H. Pehl, 1974, "Semiconductor Radiation Detectors", in Nuclear Spectroscopy and Reactions, Part A, Ed. J. Cerny, Academic Press,
[5] J. Kemmer, "Improvement of detector fabrication by the planar process".

[6] A.H. Walenta, 1987, " Principles and new developments of semiconductor radiation detectors", Nucl. Instr. and Meths, A253

[7] liro Hietanen, JukkaLindgren, Risto Orava , Tuure Tuuva, Richard Brenner, Mikael Andersson, Kari Leinonen and Hannu Ronkainen, 1991, "Ion Implanted Silicon Detectors processed on a $100 \mathrm{~mm}$ wafer", from Nuclear Instruments and Methods in Physics Research,

[8] Michael Shur, 1990, "Physics of semiconductor devices" Prentice Hall India,

[9] E. Fretwurst et al, 1987, "Development of large area silicon detectors"

[10] Lacaita, M. Ghioni, F. Zappa, G. Ripamonti and S. Cova, 1993, "Recent Advances in the Detection of Optical Photons with Silicon Photodiodes", from Nuclear Instruments and Methods in Physics Research, A326 